\title{
A generalization of Riesz-Fischer's theorem.
}

\author{
By Ichiro AмемiyA
}

(Received Feb. 12, 1953)

L. Kantorovitch [1] has proved, generalizing Riesz-Fischer's theorem to an abstract form, that a normed semi-ordered linear space is complete if the norm satisfies the following two conditions:

(1) The norm is monotone complete, i. e., every non-decreasing and norm-bounded sequence of positive elements has the least upper bound.

(2) The norm is continuous, i. e., every non-increasing sequence of elements which is order-convergent to 0 also converges to 0 by the norm.

H. Nakano [2] has shown that the condition (2) can be weakened to the condition of semi-continuity, i. e.,

$\left(2^{\prime}\right)$ For every non-decreasing sequence of positive elements, $a_{\nu} \uparrow_{\nu-1}^{\infty}$, such that $a=\bigcup_{\nu=1}^{\infty} a_{\nu}$ exists, we have

$$
\|a\|=\sup _{\nu \geq 1}\left\|a_{\nu}\right\| \text {. }
$$

Here we shall show that even this condition $\left(2^{\prime}\right)$ is superfluous, i. e. only the condition (1) suffices for the completeness of the normed semi-ordered linear space.

First we shall prove the following

LEMMA. If a normed semi-ordered linear space $R$ has a monotone complete norm, then there exists a positive real number $\alpha \leqq 1$ such that $0 \leqq a_{\nu} \uparrow_{\nu=1}^{\infty} a$ implies always

$$
\sup _{\nu \geq 1}\left\|a_{\nu}\right\| \geq \alpha\|a\|
$$

PROOF. If we can not find such a number $\alpha$, then there exists a double sequence of positive elements $a_{\mu, \nu}$ and a sequence $a_{\mu}(\mu=1,2, \cdots)$, such that we have

$$
0 \leqq a_{\mu, \nu} \uparrow_{\nu-1}^{\infty} a_{\mu}, \quad\left\|a_{\mu}\right\| \geqq \mu \quad \text { and } \quad \sup _{\nu \geq 1}\left\|a_{\mu, \nu}\right\| \leqq \frac{1}{2^{\mu}} .
$$


Now putting

$$
b_{\nu}=a_{1, \nu}+a_{2, \nu}+\cdots+a_{\nu, \nu}
$$

for every $\nu=1,2, \cdots$, we obtain a norm bounded non-decreasing sequence and hence there exists the least upper bound $b=\bigcup_{\nu-1}^{\infty} b_{v}$, because we have

$$
\left\|b_{\nu}\right\| \leq \frac{1}{2}+\frac{1}{4}+\cdots+\frac{1}{2^{\nu}}<1 .
$$

Then we have $b \geq a_{\mu}(\mu=1,2, \cdots)$ and hence $\|b\| \geq \mu$, a contradiction.

By virtue of this lemma, we can see easily that for any sequence of elements $a_{\nu}(\nu=1,2, \cdots)$ such that $\left\|a_{\nu}\right\| \leqq \frac{1}{2^{\nu}}$, the series $a_{1}+a_{2}+\cdots$ is convergent to the same sum both in order and in norm. Hence we can select from any Cauchy sequence a subsequence $a_{\nu}(\nu=1,2, \cdots)$ such that $\nu \leqq \mu, \rho$ implies $\left\|a_{\mu}-a_{\rho}\right\| \leqq \frac{1}{2^{\nu}}$; then $a=a_{1}+\left(a_{2}-a_{1}\right)+\left(a_{3}-a_{2}\right)+\cdots$ is the limit of $a_{\nu}$ by norm-topology. Thus we have:

THEOREM. If the norm of a normed semi-ordered linear space $R$ is monotone complete, then $R$ is complete.

REMARK. This theorem is also valid in the case that the topology of $R$ is sequential and compatible with the order, i. e., as a basis of the neighbourboods of 0 we can take $V_{\nu}(\nu=1,2, \cdots)$ such that

$$
x \in V_{\nu} \text { and }|x| \geqq|y| \text { imply } y \in V_{\nu} .
$$

Here the monotone completeness of the topology is defined similarly as in (1) ; we have only to replace the word " norm-bounded" by "topologically bounded".

\section{Department of Mathematics, Hokkaido University}

\section{References}

[1] L. Kantorovitch, Linear halbgeordnete Räume, Recueil Math., 44 (1937), pp. 121-165.

[2] H. Nakano, Modulared semi.ordered linear spaces, 1950, Tokyo Math. Book Series vol. 1, Theorem 30. 17. 